\title{
Enhanced black state induced by spatial silver nanoparticles in an electrochromic device
}

\author{
Ki Ryong Jeong ${ }^{1,3}$, Illhwan Lee ${ }^{1,3}$, Jae Yong Park ${ }^{1}$, Chung Sock Choi ${ }^{2}$, Sang-Hwan Cho² and Jong-Lam Lee ${ }^{1}$
}

The use of three-dimensional (3D) hierarchical indium tin oxide (ITO) branches of electrochromic devices (ECDs) is an effective approach for increasing the optical properties via localized surface plasmon resonance compared with two-dimensional nanostructured electrodes. ECDs with 3D branches were designed to operate in transparent, mirror and black states.

Finite-difference time-domain simulation was used to find the electrical field distributions in three types of ECD: glass/ITO with $\mathrm{Ag}$ film, glass/ITO branches and glass/ITO branches with Ag nanoparticles. The ECDs had an optical transmittance of $73.76 \%$ in the transparent state, a reflectance of $79.77 \%$ in the mirror state and a reflectance of $8.78 \%$ in the black state. We achieved an ECD with high stability that can show 10000 switching cycles among the three states.

NPG Asia Materials (2017) 9, e362; doi:10.1038/am.2017.25; published online 17 March 2017

\section{INTRODUCTION}

Electrochromic devices (ECDs) can exhibit reversible color changes induced by electric energy and the resulting electrochemical redox reactions of materials. ${ }^{1,2}$ The changes in optical states are consequences of a change in the electronic state as a result of electron transfer between the electrochromic (EC) material and an electrode. ECDs offer many advantages over conventional displays, including a low operating voltage $\left(V_{\mathrm{OP}}\right)$, memory effects, color variations and visibility in sunlight. ${ }^{3-7}$ Therefore, ECDs are expected to achieve applications in information displays or in light-modulating devices such as smart windows, switchable mirrors, electronic papers and chemical sensors. ${ }^{8-12}$

Conventional ECDs are composed of a non-metal (NM) EC material (for example, poly(ethylene oxide), poly(methyl methacrylate), polyvinylidene difluoride, $\left.\mathrm{WO}_{3}, \mathrm{MoO}_{3}, \mathrm{Ir}(\mathrm{OH})_{3}, \mathrm{NiO}\right){ }^{13-17}$ In particular, $\mathrm{WO}_{3}$, which is the most widely known EC material, has attracted considerable attention because of its broad applications such as in ECDs, photocatalysis and sensing devices. ${ }^{3}$ However, the use of NM-ECD encounters two drawbacks. (1) It has low optical transmittance when in the transparent state because of the inherent color and high extinction coefficient $(k)$ of the NM materials compared with the metal ions in the electrolyte of the transparent state. ${ }^{13-18}$ (2) The NM-ECD cannot exhibit a mirror state. Because free electrons are rarely generated in NM materials, an electric field easily penetrates the NM materials. Therefore, most of the incident energy is absorbed or transmitted. As a result, NM-ECDs are not suitable for use in displays and windows.

Silver (Ag) has been used as an EC material because of its superior optical properties. Because Ag readily assembles into nanoparticles
(NPs), an ECD with Ag (Ag-ECD) can exhibit a transparent state (average optical transmittance $T_{\text {avg }}>75 \%$ ) and a mirror state. ${ }^{19-21}$ It can also achieve a black state if it is combined with an indium tin oxide (ITO) sol-gel nanostructured electrode, but these Ag-ECDs have high average optical reflectance values $\left(R_{\mathrm{avg}}>10 \%\right)$ in the visible spectrum, ${ }^{19,20}$ possibly as a result of weak plasmon resonance of the $\mathrm{Ag}$ NPs on the ITO sol-gel nanostructured electrode. ${ }^{22-26}$ Another problem is that the ITO sol-gel nanostructure reacts with the electrolyte, causing the Ag-ECD to degrade during repeated cycling. ${ }^{19,20,27,28}$ Such problems could be overcome by spatially separating the Ag NPs to favor localized surface plasmon resonance (LSPR) between them, thereby maximizing the absorption of photons. ${ }^{29,30}$

Here, we report a way to use spatially separated Ag NPs to obtain a completely black state in an ECD. Three-dimensional (3D) ITO branches were used as scaffolds to obtain spatially separated Ag NPs to achieve LSPR between them in the visible spectrum. The 3D ITO branches were prepared by using electron beam (e-beam) evaporation on a glass/ITO substrate. ${ }^{31}$ EC materials were inserted between the flat ITO and 3D ITO branches that were separated by a $200 \mu \mathrm{m}$ thick polyimide film. When a positive voltage was applied to the flat ITO, $\mathrm{Ag}^{+}$ions in the electrolyte precipitated on the surface of the 3D ITO branches to yield spatially separated Ag NPs. The Ag NPs can effectively interact with incident photons to produce LSPR; this reaction led to a completely black state by totally suppressing the reflection of incident light. The completely black state induced by LSPR was explained in a theoretical analysis using the finite-difference time-domain (FDTD) method. Such an ECD is expected to be useful when used together with an organic

${ }^{1}$ Department of Materials Science and Engineering, Pohang University of Science and Technology (POSTECH), Pohang, South Korea and ${ }^{2}$ Samsung Display Company Limited, Youngin, Gyeonggi, South Korea

${ }^{3}$ These authors contributed equally to this work.

Correspondence: Professor J-L Lee, Department of Materials Science and Engineering, Pohang University of Science and Technology (POSTECH), 77 Cheongam-Ro, Nam-Gu, Pohang, Gyeongbuk 790-784, South Korea.

E-mail: jllee@postech.ac.kr

Received 22 June 2016; revised 26 November 2016; accepted 28 November 2016 
light-emitting diode (OLED)-based transparent display, in which a black state is hard to define on its own; if it is stacked behind the transparent OLED, the transparent OLED in the OFF state will look black when the ECD is switched to its black state.

\section{EXPERIMENTAL PROCEDURES}

\section{Materials}

Silver nitrate $\left(\mathrm{AgNO}_{3}\right)$ as the electrochromic material, copper chloride $\left(\mathrm{CuCl}_{2}\right)$ as the electrochemical mediator, tetra- $n$-butylammonium bromide (TBABr) as the supporting electrolyte, poly(vinyl butyral) (PVB) as the host polymer and dimethyl sulfoxide (DMSO) as the solvent were purchased from both Samchun Chemical (Pyeongtaek, South Korea) and Sekisui (Tokyo, Japan).

\section{Fabrication of ITO branch electrodes}

An ITO branch electrode on glass coated with ITO (150 nm thick, $\sim 10 \Omega \mathrm{sq}^{-1}$ ) was fabricated by conventional e-beam evaporation. The ITO pellets used to obtain ITO branches via evaporation were made of indium oxide powder $(99.99 \%$ doped with $10 \%$ tin, from Mining \& Chemical Products, Wellingborough, UK) after sintering at $1100{ }^{\circ} \mathrm{C}$ for $24 \mathrm{~h}$ in ambient atmosphere. The ITO branch electrode was grown by evaporating these pellets at a rate of $1 \mathrm{~nm} \mathrm{~s}^{-1}$. The chamber pressure of the e-beam evaporator was $\sim 10^{-4}$ Torr during deposition, and the substrate temperature was $350^{\circ} \mathrm{C}$.

\section{Fabrication of ECDs}

An electrolyte solution was prepared by dissolving $0.05 \mathrm{mmol}$ of $\mathrm{AgNO}_{3}$, $0.06 \mathrm{mmol}$ of $\mathrm{CuCl}_{2}$ and $1.56 \mathrm{mmol}$ of TBABr in $5 \mathrm{ml}$ of DMSO with $5.5 \mathrm{~g}$ of
PVB as the host polymer. A flat ITO electrode was cleaned sequentially with acetone, isopropyl alcohol and deionized water for $3 \mathrm{~min}$ each. The prepared electrolyte was coated onto the flat ITO electrode with a $200 \mu \mathrm{m}$ thick polyimide spacer (Youngwoo Trading, Seongnam, South Korea) and a device size of $1 \mathrm{~cm} \times 1 \mathrm{~cm}$ and then assembled with the ITO branch electrode.

\section{Measurement}

The surface structure and morphology analyses on the two electrodes were performed using a scanning electron microscope system (PHILIPS XL 30S, FEI Company, Eindhoven, Netherlands) at an accelerating voltage of $5 \mathrm{kV}$ and a working distance of $6 \mathrm{~mm}$. The $T_{\text {avg }}$ and $R_{\text {avg }}$ of the ECD and ITO branch electrodes were measured using a ultraviolet-visible/near-infrared (UV-VisNIR) spectrophotometer (Carry 5000, Agilent, Santa Clara, CA, USA). The ECD was operated using a Keithley 2400 source measurement unit (Keithley, Cleveland, OH, USA). Scattering profiles and dark-field reflectance images were measured using a dark-field microscopy system with a true-color-imaging charge-coupled device camera and a spectrometer (OLYMPUS BX 51, Olympus, Tokyo, Japan). A stability test was performed using a multichannel electrochemical analyzer (IVIUM TECH, Eindhoven, Netherlands). Highresolution transmission electron microscopy images were obtained using a Cs-corrected JEM 2200FS (JEOL, Tokyo, Japan) operated at $200 \mathrm{kV}$.

\section{Theoretical modeling and calculation}

Two-dimensional FDTD simulations were performed to explain the effect of the LSPR of Ag NPs on the ITO branches. The simulation tool was a commercial electromagnetic calculation module ( $\mathrm{R}$ Soft Full wave) of R soft 2014.09 (Synopsys, Mountain View, CA, USA and R soft Design Group, Inc.,

a

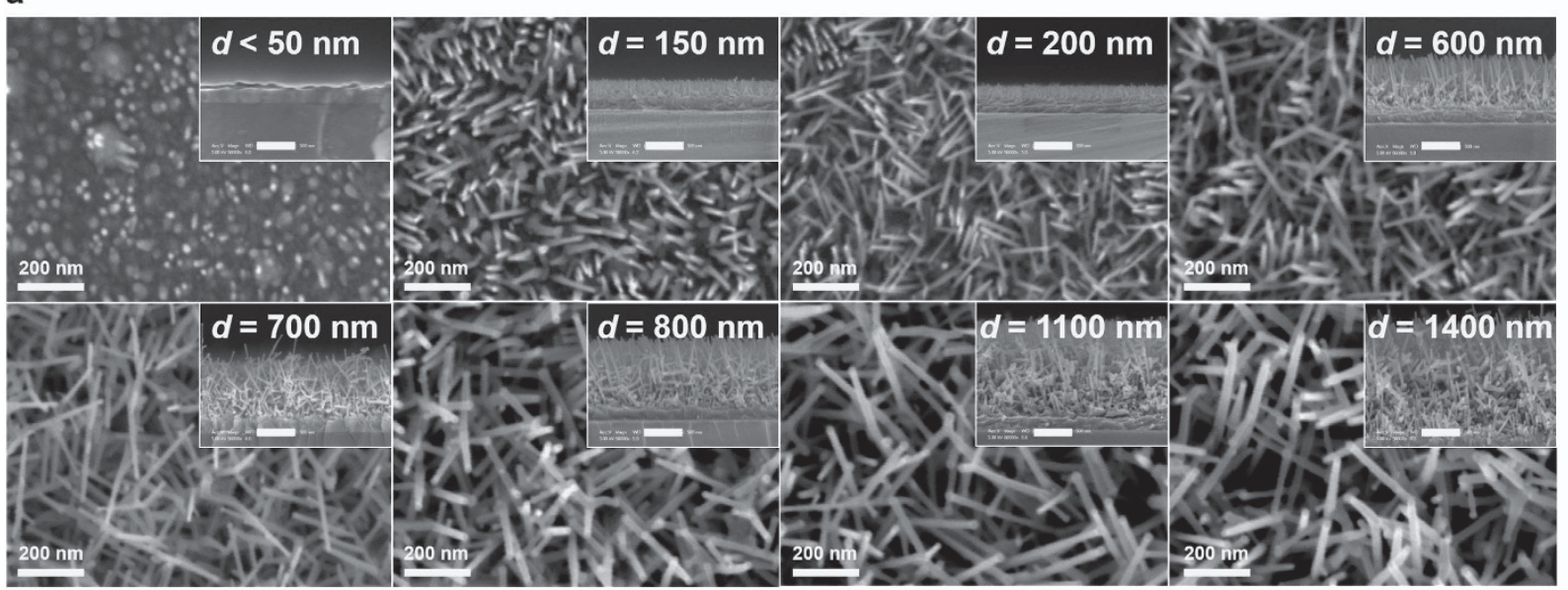

b

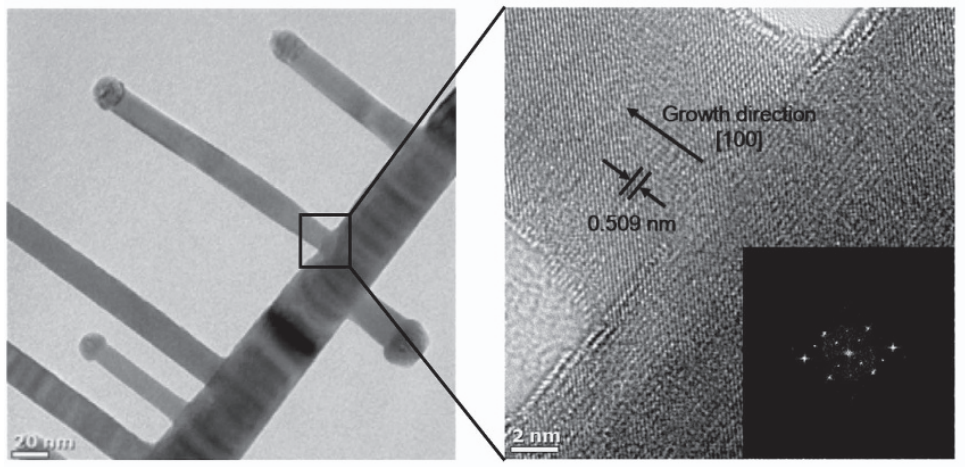

C

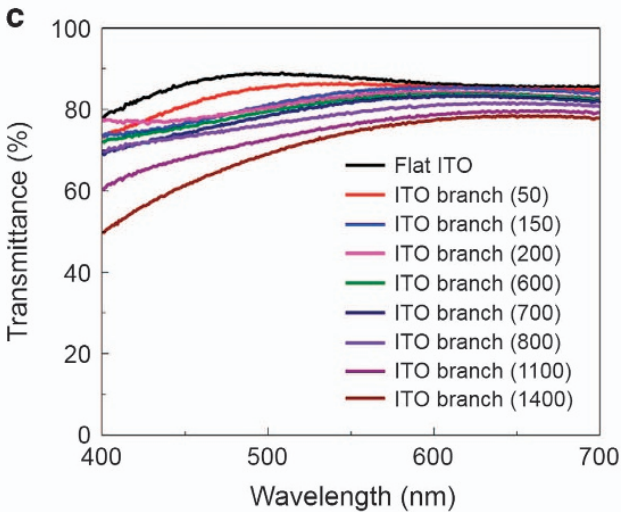

Figure 1 Characterization of indium tin oxide (ITO) branches. (a) Top-view and cross-sectional scanning electron microscope (SEM) images of different thickness achieved by electron beam evaporation. Scale bar, $500 \mathrm{~nm}$. (b) High-resolution transmission electron microscope (TEM) images and electron diffraction pattern (inset) indicate well-crystallized ITO with (100) orientation (lattice constant: $d=0.509 \mathrm{~nm}$ ). (c) Transmittance in the visible spectrum $(400 \leqslant \lambda \leqslant 700 \mathrm{~nm})$ of ITO branches of a range of thickness grown on the flat ITO ( $\lambda$ : wavelength). 
Table 1 Optical properties

ITO branch thickness (nm)

\begin{tabular}{lccccccrrr} 
Optical characteristics & 0 & 50 & 150 & 200 & 600 & 700 & 800 & 1100 & 1400 \\
\hline Electrodes (\%) & 86.2 & 83.9 & 81.82 & 81.48 & 80.46 & 79.48 & 77.84 & 74.32 & 70.86 \\
Transparent state (\%) & 80.75 & 78.05 & 74.41 & 74.16 & 73.85 & 73.76 & 70.72 & 68.44 & 65.54 \\
Mirror state (\%) & 79.55 & 78.14 & 78.92 & 78.94 & 79.48 & 79.77 & 78.85 & 79.69 & 79.44 \\
Black state (\%) & 56.23 & 38.8 & 11.39 & 9.85 & 8.16 & 8.78 & 8.6 & 8.62 & 8.92 \\
\hline
\end{tabular}

Abbreviation: ITO, indium tin oxide.

Measured characteristics of ITO branch electrodes and the three states of electrochromic device (ECD) as a function of ITO branch thickness. Values for electrodes and transparent state correspond to average transmittance and those for mirror and black states are average reflectance. Wavelength range of average transmittance and reflectance is from 400 to $700 \mathrm{~nm}$.

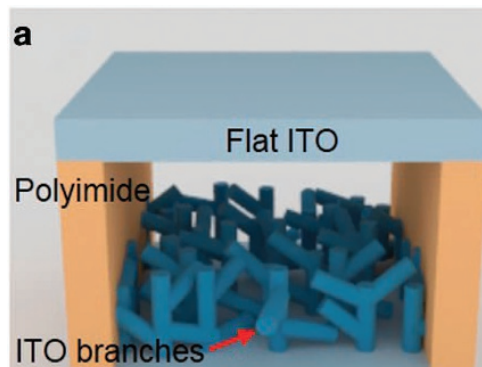

Flat ITO b

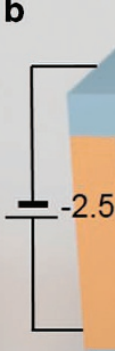

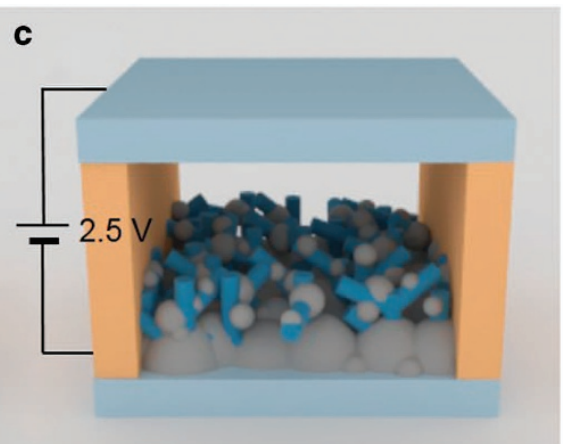
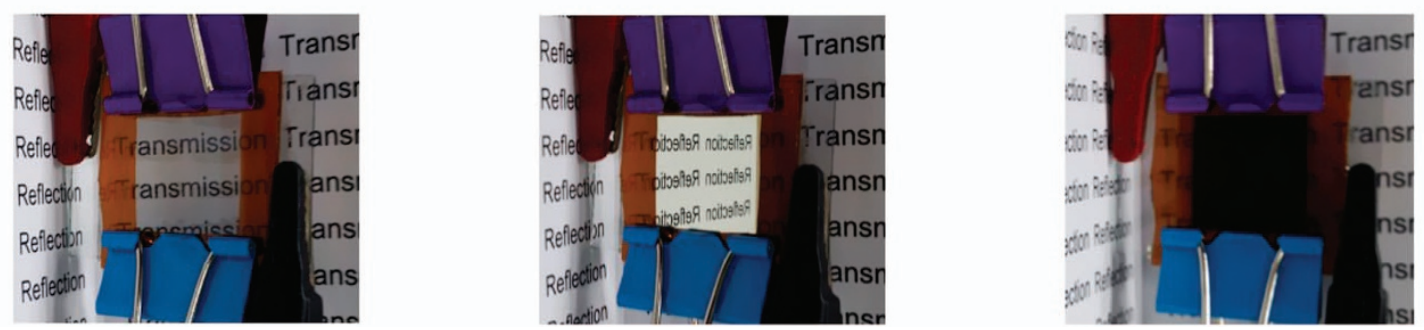

Figure 2 Operating states of electrochromic device (ECD). Schematics (top) and photographs (bottom) of the ECD that consists of the flat indium tin oxide (ITO) and ITO branches. Active area of device is $1 \mathrm{~cm} \times 1 \mathrm{~cm}$. (a) Transparent, (b) mirror and (c) black states.

Ossining, NY, USA). The boundary condition was set to a perfectly matched layer to avoid unwanted reflection at the edge of the structures. The simulation structures consisted of glass/ITO with Ag film, glass/ITO branches and glass/ ITO branches with Ag NPs. The heights of the main nanorods and secondary nanorods of ITO branches were fixed at 300 and $150 \mathrm{~nm}$, respectively. The diameters of ITO nanorods and $\mathrm{Ag}$ nanodots were fixed at 30 and $20 \mathrm{~nm}$, respectively. The grid size of the simulation was set to $2 \mathrm{~nm}$ to resolve the $\mathrm{Ag}$ NPs. The top of the device was illuminated continuously with A plane wave with $\lambda=420 \mathrm{~nm}$. During the illumination, both electric and magnetic fields were calculated alternately time-step by time-step. From this calculation, the electric field distributions $\mathrm{E}(\vec{r}, t)$ were recorded as a function of time by a discrete Fourier transform monitor. Then, the electric fields were converted to light absorption:

$$
U_{\text {abs }}(\vec{r}, t)=4 \pi n k|E(\vec{r}, t)|^{2} / \lambda
$$

where $n$ is the refractive index. The calculation was performed until the light had propagated $20 \mu \mathrm{m}$ in free space to reach the steady state.

\section{RESULTS AND DISCUSSION}

\section{Fabrication of ITO branches}

The self-assembled single-crystalline ITO branches were grown in an e-beam evaporator. ${ }^{32}$ At an oxygen partial pressure of $10^{-4}$ Torr, e-beam irradiation can decompose ITO to indium, tin and oxygen.
Then, the evaporated metals form tin-doped indium nanodots that self-catalyze a vapor-liquid-solid process to generate nanostructures and produce branched 3D ITO structures. To identify the morphology of the 3D ITO branches, we obtained top-view scanning electron microscope images as a function of their thickness $\left(t_{\mathrm{ITO}}\right)$ (Figure 1a). At the beginning of ITO growth $\left(t_{\text {ITO }}<50 \mathrm{~nm}\right)$, nanodot shapes were observed. As $t_{\text {ITO }}$ increased, a branched hierarchical structure developed and became crosslinked. Therefore, the morphology of ITO branches can be controlled by adjusting the parameters of the e-beam evaporation process. High-resolution transmission electron microscopy confirmed the structure of the ITO branches (Figure 1b). At the junctions between branches, the continuous atomic arrangement with a $\{100\}$ family plane orientation of cubic-based bixbyite $\mathrm{In}_{2} \mathrm{O}_{3}$ could be confirmed. Because the $\mathrm{In}_{2} \mathrm{O}_{3}\{100\}$ family planes have metallic indium terminations, the metallic self-catalyst (tin-doped indium nanodots) can form easily at these planes. ${ }^{33}$ The fabricated ITO branches show a well-aligned bixbyite singlecrystal $\mathrm{In}_{2} \mathrm{O}_{3}$ (100) orientation with a lattice spacing of $0.509 \mathrm{~nm}$. The optical properties were affected by $t_{\text {ITO }}$ (Table 1). Over the visible spectrum $(400 \leqslant \lambda \leqslant 700 \mathrm{~nm}$ ) (Figure 1c), the ITO branches had $T_{\text {avg }}=83.9 \% \quad\left(t_{\mathrm{ITO}}=50 \mathrm{~nm}\right), 79.48 \% \quad\left(t_{\mathrm{ITO}}=700 \mathrm{~nm}\right)$ and $70.86 \%$ $\left(t_{\mathrm{ITO}}=1400 \mathrm{~nm}\right)$. For comparison, the flat ITO (that is, $\left.t_{\mathrm{ITO}}=0 \mathrm{~nm}\right)$ 
had $T_{\mathrm{avg}}=86.2 \%$. The slight reduction in the $T_{\mathrm{avg}}$ of the ITO branch samples may be a result of light absorption and reflection by the metallic self-catalyst.

\section{Operation of ECD with ITO branches}

The ECD was fabricated by sandwiching the electrolyte between two transparent electrodes separated by a polyimide spacer (interelectrode distance of $200 \mu \mathrm{m}$ ). The electrolyte was a transparent and colorless solution of $\mathrm{AgNO}_{3}, \mathrm{CuCl}_{2}$, tetra- $n$-butylammonium bromide (TBABr), PVB and DMSO. The working electrode was the flat ITO; the counter electrode was composed of $3 \mathrm{D}$ ITO branches with $t_{\mathrm{ITO}}=700 \mathrm{~nm}$. Transparent, mirror and black states were achieved in a single device (Figure 2). In the transparent state, $\mathrm{Ag} \mathrm{NO}_{3}$ was dissolved in the electrolyte solution (Figure 2a). Application of $-2.5 \mathrm{~V}$ to the flat ITO caused electrochemical reduction of dissolved $\mathrm{Ag}^{+}$to $\mathrm{Ag}^{0}$ that precipitated on the flat ITO to form a mirror state (Figure $2 \mathrm{~b}$ ). As the potential was swept from -2.5 to $0.5 \mathrm{~V}$, the electrodeposited $\mathrm{Ag}$ NPs oxidized to $\mathrm{Ag}^{+}$that dissolved; thus, the ECD became transparent again. Application of $+2.5 \mathrm{~V}$ to the ITO branches caused electrochemical reduction of $\mathrm{Ag}^{+}$to $\mathrm{Ag}$ NPs and resulted in a nonreflective black state (Figure 2c). The transparent state was recovered by applying an oxidation voltage of $-0.5 \mathrm{~V}$ to dissolve the Ag NPs. Therefore, the transitions among the transparent, mirror and black states were reversible. $V_{\mathrm{OP}}= \pm 2.5 \mathrm{~V}$ was chosen because it was the best at producing black and mirror states (Table 2 and Supplementary Figure S1).

\section{Optical properties of ECD with ITO branches}

The $T_{\text {avg }}$ of the transparent state and $R_{\text {avg }}$ of both the mirror and black states were measured at $0 \leqslant t_{\text {ITO }} \leqslant 1400 \mathrm{~nm}$ (Figure $3 \mathrm{a}$ and Table 1 ). In the reference $\mathrm{ECD}\left(\mathrm{ECD}_{\text {ref }}\right)$, flat ITO was used in both electrodes $\left(t_{\mathrm{ITO}}=0 \mathrm{~nm}\right)$. The $T_{\mathrm{avg}}$ of the transparent state was $80.75 \%$ in the $\mathrm{ECD}_{\text {ref }}$ but gradually decreased to $\sim 65.54 \%$ at $t_{\mathrm{ITO}}=1400 \mathrm{~nm}$ because of the introduction of ITO branches. At $50 \leqslant t_{\mathrm{ITO}} \leqslant 700 \mathrm{~nm}$, $T_{\text {avg }}$ was $>73 \%$ but decreased to $65.54 \%$ at $t_{\mathrm{ITO}}=1400 \mathrm{~nm}$. This trend indicates that the decreased $T_{\text {avg }}$ was induced by high $k$ that is related to the decay of the incident electric field by a factor of $e^{-\alpha t}$ according to the Beer-Lambert law (absorption coefficient, $\alpha=4 \pi k / \lambda)$ as $t_{\text {ITO }}$ increased. In the mirror state, electrochemical reduction of $\mathrm{Ag}^{+}$occurred at the surface of the flat ITO, and therefore $R_{\text {avg }}$ was independent of $t_{\mathrm{ITO}}$. The formation of Ag NPs on the flat ITO generates electrons that block the electric field such that it does not penetrate the NPs. Therefore, the incident energy is not absorbed or transmitted, resulting in reflectivity that is good regardless of $t_{\text {ITO. }}$ In the black state, $R_{\mathrm{avg}}$ significantly decreased from $56.23 \%$ at $t_{\mathrm{ITO}}=0 \mathrm{~nm}$ to $9.85 \%$ at $t_{\mathrm{ITO}}=200 \mathrm{~nm}$. Furthermore, at $600 \leqslant t_{\mathrm{ITO}}$ $\leqslant 1400 \mathrm{~nm}, R_{\text {avg }}$ remained at $\sim 8 \%$, and therefore a deep black state was achieved. The deepening of the black state may be a result of the deposition of Ag NPs on the 3D surface of the hierarchical nanostructure. As $t_{\mathrm{ITO}}$ increased, the height of the branched 3D structure increased, and they became crosslinked. Therefore, Ag NPs aggregated on the ITO hierarchical structure can be spatially separated, resulting in LSPR that completely suppressed light reflection.

To confirm the change of $R_{\text {avg }}$ with $t_{\text {ITO }}$ in the black state, we measured the optical reflectance spectra (Figure $3 \mathrm{~b}$ ) and transmittance spectra (Supplementary Figure S2) of the ECD $\mathrm{Eef}_{\text {ref }}$ and the ECD with $t_{\mathrm{ITO}}=700 \mathrm{~nm}\left(\mathrm{ECD}_{700}\right)$. In the mirror state, $R_{\mathrm{avg}}$ was $79.55 \%$ in the $\mathrm{ECD}_{\text {ref }}$ and $79.77 \%$ in the $\mathrm{ECD}_{700}$; in the black state, $R_{\text {avg }}$ was $56.23 \%$ in the $\mathrm{ECD}_{\text {ref }}$ and $8.78 \%$ in the $\mathrm{ECD}_{700}$; and in the transparent state, it was $8.87 \%$ in the $\mathrm{ECD}_{\text {ref }}$ and $8.36 \%$ in the $\mathrm{ECD}_{700}$. In
Table 2 Operating voltage

Operating voltage $(V)$

\begin{tabular}{lccccccc} 
Optical characteristics & $|0.5|$ & $|1.7|$ & $|2.0|$ & $|2.3|$ & $|2.5|$ & $|2.6|$ & $|2.7|$ \\
\hline Mirror state (\%) & 8.81 & 8.8 & 33.96 & 71.73 & 79.77 & Degradation \\
Black state (\%) & 18.98 & 18.72 & 62.15 & 80.96 & 88.57 &
\end{tabular}

The average reflectance and absorbance in the visible spectrum $(400 \leqslant \lambda \leqslant 700 \mathrm{~nm})$ of mirror and black states as a function of the operating voltage. Values for mirror state correspond to average reflectance and those for black state is average absorbance. ( $\lambda$ : wavelength).

the black state, the $\mathrm{ECD}_{700}$ had an $R_{\mathrm{avg}}$ value that was lower by $47 \%$ arithmetically and $84 \%$ proportionally compared with the $\mathrm{ECD}_{\text {ref. }}$ Therefore, in practice, the $\mathrm{ECD}_{\text {ref }}$ can assume only transparent, mirror and mirror-like states that have a nonblack color, but the $\mathrm{ECD}_{700}$ can additionally assume a black state.

To measure the $R_{\text {avg }}$ of the ITO branches with Ag NPs only, without considering any other component of the ECD, we obtained ITO branches with Ag NP by separating the ECDs into two electrodes during operation in the black state and then evaporating the residual electrolyte from the surface of the ITO branches that contained Ag NPs. This sample had $R_{\mathrm{avg}}=6.0 \%$ (Supplementary Figure S3). This result indicates that the deep black state was obtained because the Ag NPs completely suppressed the reflection of incident light.

\section{Analysis of operating states}

We analyzed the surface structures of both flat ITO and ITO branches. In particular, to verify the morphologies of the Ag NPs that formed on the flat ITO and ITO branches, we used an in situ method to obtain scanning electron microscope images: when $V_{\mathrm{OP}}= \pm 2.5 \mathrm{~V}$ was applied to the ECD, Ag NPs formed on the flat ITO and ITO branches. Then, we separated the ECD into two different electrodes with Ag NPs and removed the residual solution by evaporation. The flat ITO without Ag NPs had a smooth surface (Figure 4a); when Ag NPs were deposited on this surface, they connected to each other to form a planar deposit similar to a Ag film. Such uniform nucleation of the Ag NPs would be greatly facilitated on the flat ITO and result in the mirror state. In contrast, the ITO branches without Ag NPs had 3D structures that were linked and branched, resulting in a highly rough $3 \mathrm{D}$ surface (Figure $4 \mathrm{~b}$ ). This branched $3 \mathrm{D}$ structure can be attributed to the spatial separation of the aggregated Ag NPs when they are deposited on the ITO branches. Because the Ag NPs do not connect to each other, the surface is rough and nonreflective; therefore, the Ag NPs on the ITO branches achieved the black state by completely suppressing reflectance.

To clarify the interactions between the light and the surface morphology of the electrodes, we obtained dark-field reflectance images of both the flat ITO and ITO branches with and without Ag NPs (Figure 4c). The dark-field images of the ITO branches were brighter than those of the flat ITO with and without Ag NPs because the rough surfaces of the ITO branches collect scattered light. Furthermore, when the Ag NPs that have aggregated on the ITO branches are spatially separated, the brightness of dark-field images increased because of multiple scattering and to absorption of light by LSPR. ${ }^{34-36}$ To investigate the existence of LSPR in the Ag NPs in each sample, we also measured Rayleigh scattering profiles (Figure $4 \mathrm{~d}$ ). Over the range of $400 \leqslant \lambda \leqslant 600 \mathrm{~nm}$, in which LSPR is active, $^{37-39}$ ITO branches with Ag NPs showed intense scattering; ITO branches without Ag NPs showed relatively weak scattering, and flat ITO both with and without Ag NPs lacked the specific peaks that originate from LSPR. 

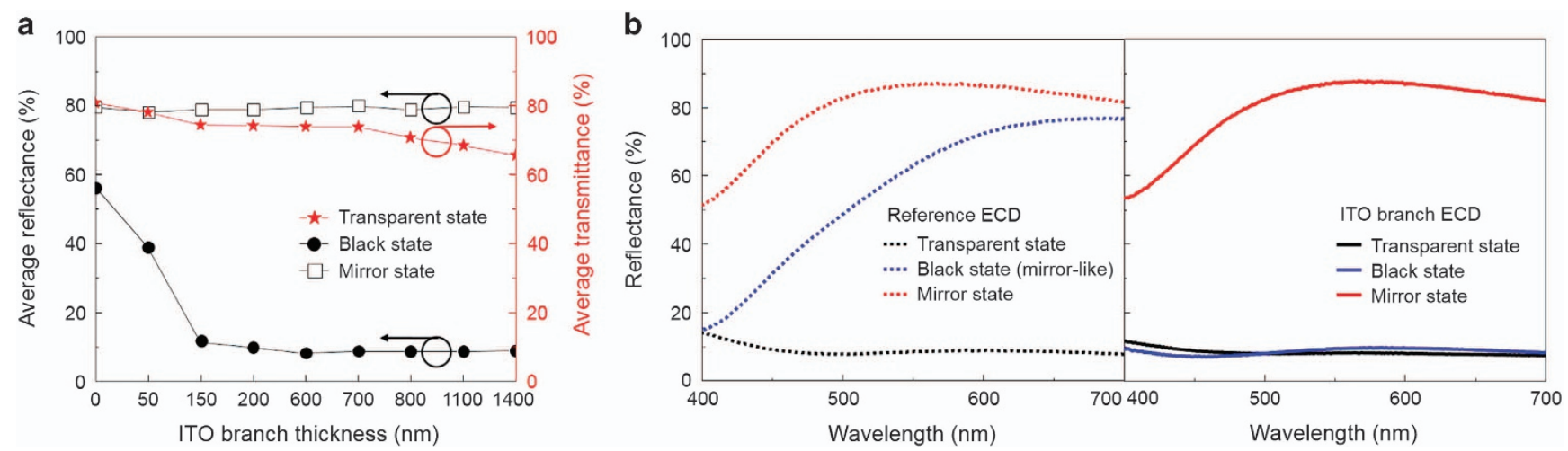

Figure 3 Optical properties of electrochromic device (ECD) with indium tin oxide (ITO) branches. (a) Average transmittance in the visible spectrum (transparent state) and average reflectance (mirror and black states) of the ECD with ITO branches as a function of their thickness. Wavelength range of average transmittance and reflectance is from 400 to $700 \mathrm{~nm}$. Reflectance spectra of (b) reference ECD without ITO branches and the ECD with $700 \mathrm{~nm}$ thick ITO branches.

a

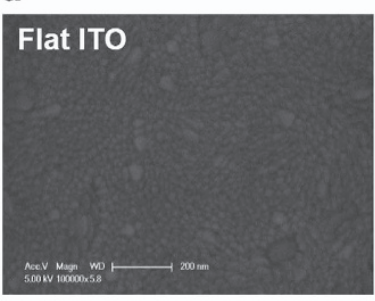

C

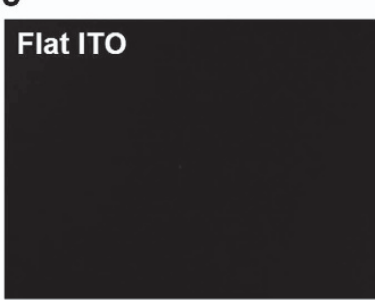

b
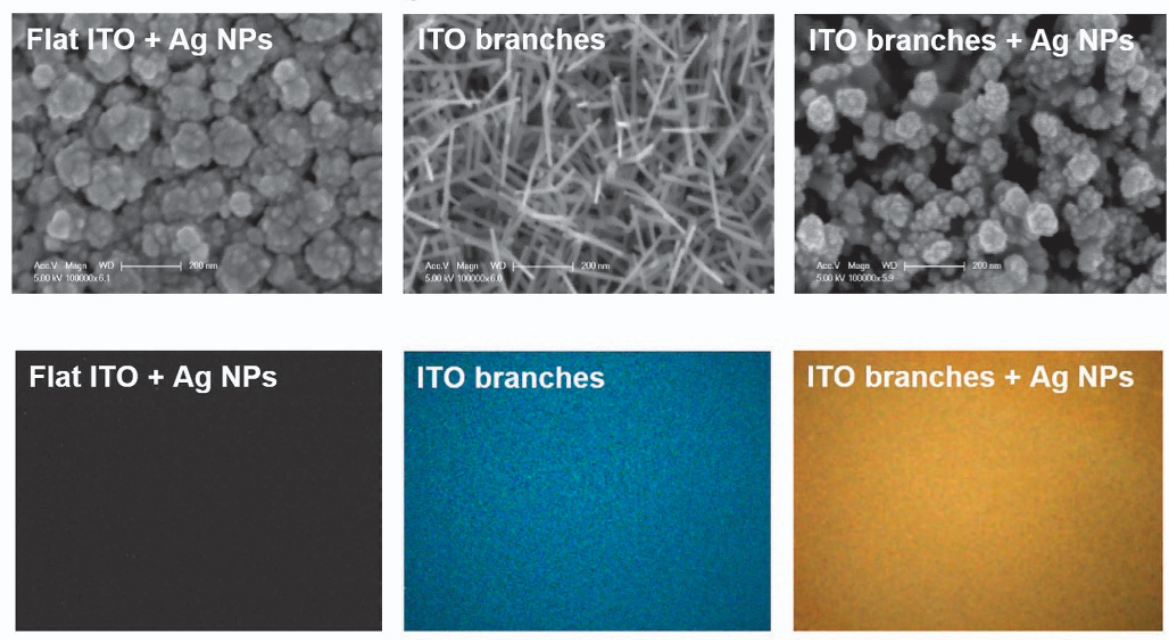

d

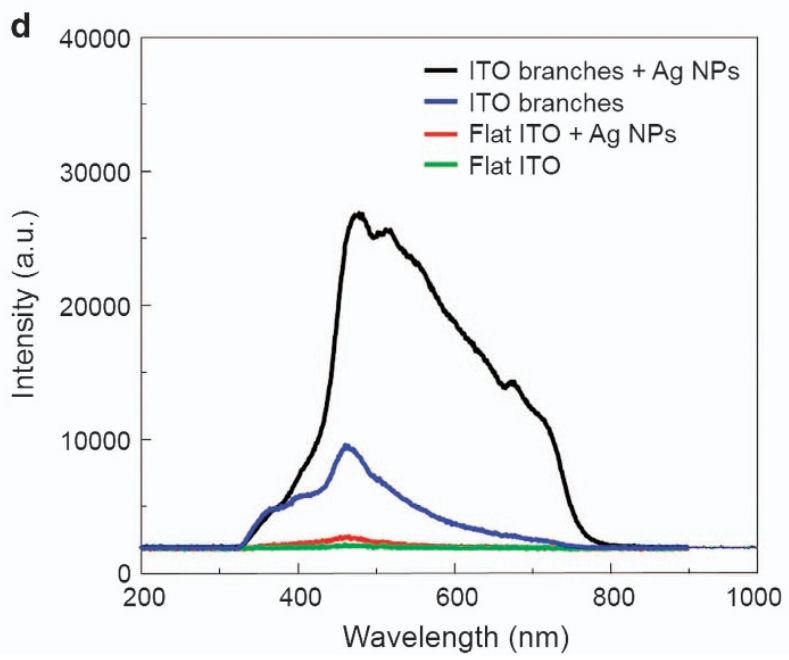

Figure 4 Analysis of surface morphologies and structures. Top-view scanning electron microscope (SEM) images of (a) flat indium tin oxide (ITO) and flat ITO with silver nanoparticles (Ag NPs), (b) ITO branches and ITO branches with Ag NPs. (c) Representative dark-field reflectance images and (d) Rayleigh scattering spectra of two transparent electrodes, one with Ag NPs and one without Ag NPs. 

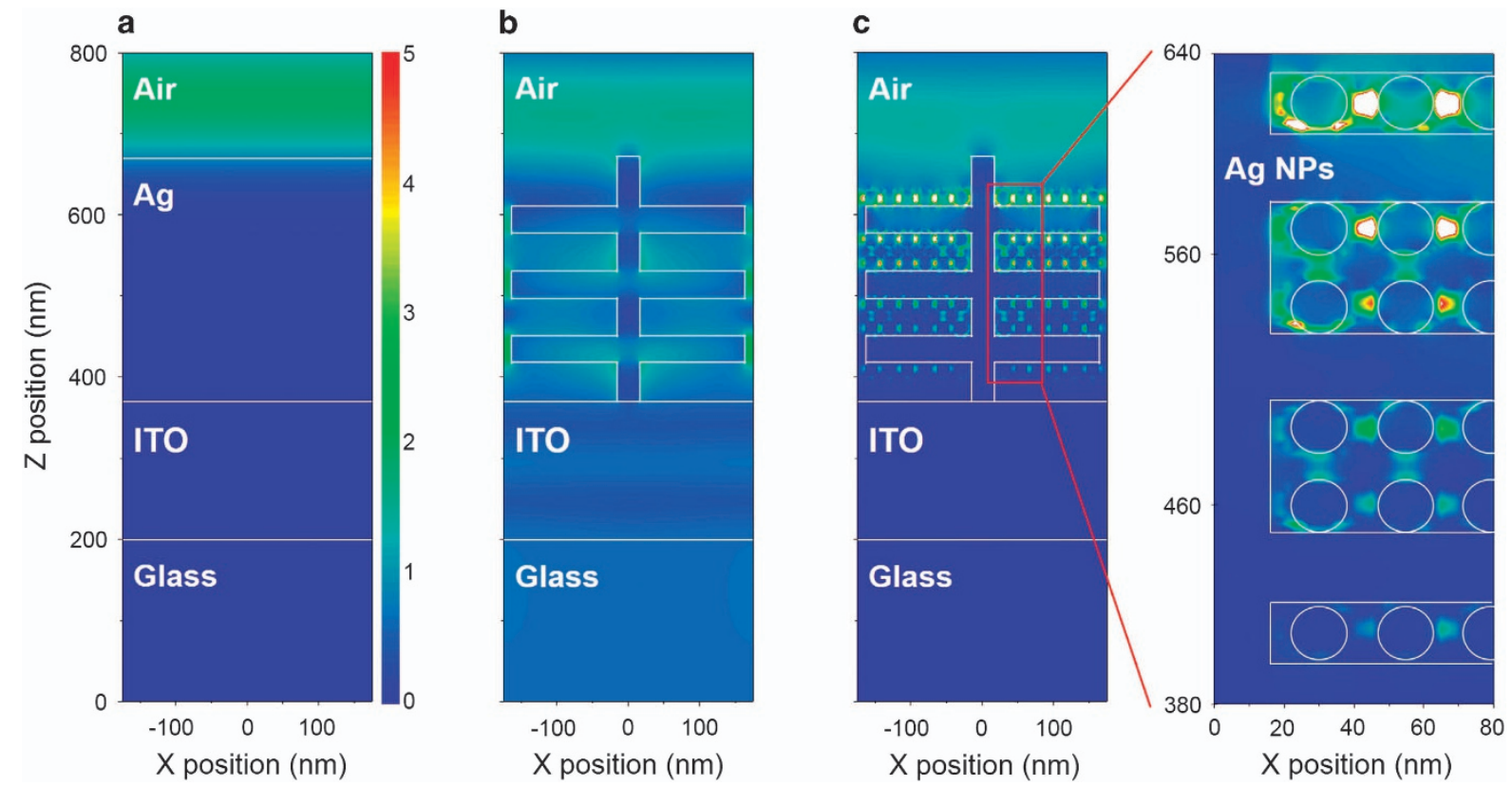

Figure 5 Finite-difference time-domain (FDTD) simulations. The $x-z$ plane cross-section of electric field distribution at wavelength $(\lambda)=420 \mathrm{~nm}$ for glass/ indium tin oxide (ITO) with (a) silver (Ag) film, (b) ITO branches and (c) ITO branches with Ag nanoparticles (Ag NPs).
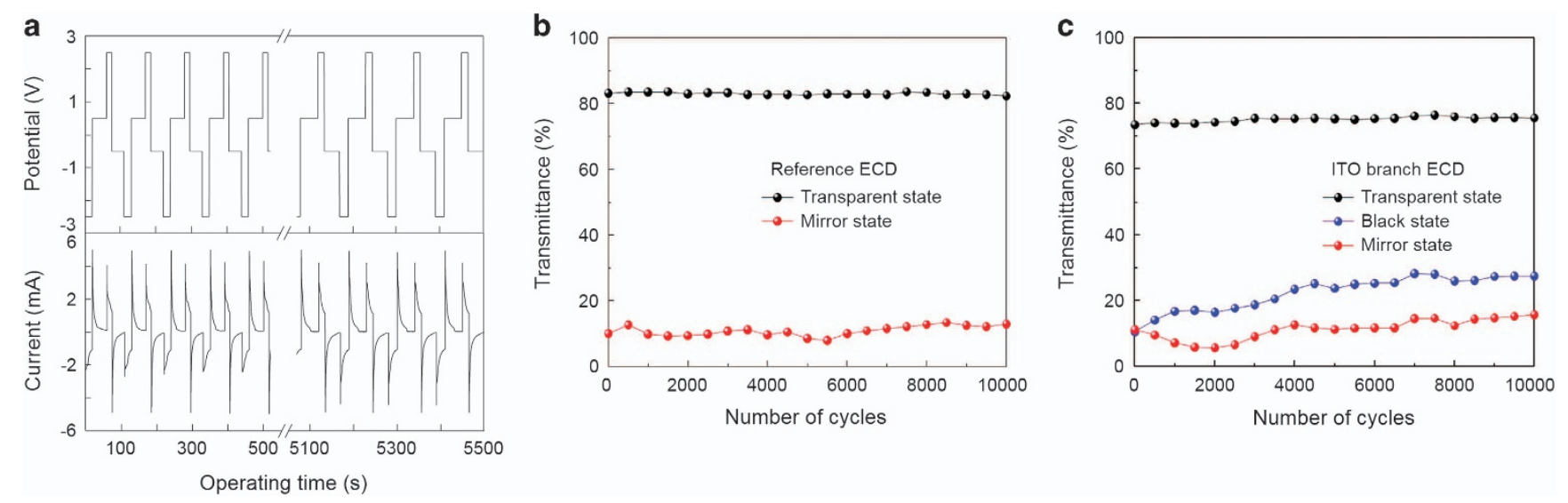

Figure 6 Repetitive operation of three states. (a) Operating of the device by sequential application of various biases. Stability test of (b) reference electrochromic device (ECD) without indium tin oxide (ITO) branches and (c) ECD with $700 \mathrm{~nm}$ thick ITO branches, switching among transparent, black and mirror states. Transmittance changes were measured at wavelength $(\lambda)=600 \mathrm{~nm}$.

\section{FDTD simulations}

To verify the origin of the low reflectance and high absorption of the black state, we conducted FDTD simulations of the $\mathrm{x}-\mathrm{z}$ plane cross-section of the electric field distribution in glass/ITO with Ag film, glass/ITO branches and glass/ITO branches with Ag NPs. In the flat ITO with Ag film, the electric field distribution did not change at the specific location, and the transmitted wave was weak at the interface between the Ag film and air (Figure 5a); the metallic Ag film on the flat ITO reflected light, resulting in the development of the mirror state. In the ITO branches without Ag NPs, the electric field was distributed evenly in all regions, and therefore the transparent state was developed (Figure 5b); this observation indicates that the generated electromagnetic wave can freely propagate without specific absorption and scattering. In the ITO branches with Ag NPs, most of the absorption of incident light occurred near the top, and therefore the field intensity in the Ag NPs was stronger near the top of the
ITO branches than at the bottom (Figure 5c). A strong field intensity was highly localized between the Ag NPs in both the horizontal and vertical directions; this distribution shows that precipitation of Ag NPs on the surface of the 3D ITO branches is required for LSPR to occur. This result indicates that the 3D ITO branches spatially separated the Ag NPs, thereby inducing effective light trapping, that in turn resulted in LSPR and caused specific absorption and scattering with weak propagation. As a result, the strong LSPR confined the electromagnetic energy and caused the formation of a deep-black state with low reflectance and high absorption.

\section{Stability of ECD}

We conducted a repetition of stability test for both the $\mathrm{ECD}_{\text {ref }}$ and $\mathrm{ECD}_{700}$. We switched among the three operating states by sequentially applying $-2.5 \mathrm{~V}(20 \mathrm{~s}), 0.5 \mathrm{~V}(40 \mathrm{~s}), 2.5 \mathrm{~V}(15 \mathrm{~s})$ and $-0.5 \mathrm{~V}(35 \mathrm{~s})$ (Figure $6 \mathrm{a}$ and Table 3 ). We also measured the switching times 
Table 3 Changes of operating current

\begin{tabular}{lcrrr}
\hline & \multicolumn{4}{c}{ Operating time (s) } \\
\cline { 2 - 5 } Current density $\left(\mathrm{mA} \mathrm{cm}^{-2}\right)$ & 100 & 500 & 5000 & 5500 \\
\hline Mirror (-2.5 V) & -1.095 & -1.081 & -1.061 & -1.069 \\
Transmission (+0.5 V) & 0.088 & 0.048 & 0.019 & 0.02 \\
Black (+2.5 V) & 1.23 & 1.168 & 1.114 & 1.125 \\
Transmission (-0.5 V) & -0.033 & -0.038 & -0.036 & -0.038 \\
\hline
\end{tabular}

Repetition of the test of the stability of operating current of electrochromic device (ECD) with $700 \mathrm{~nm}$ thick indium tin oxide (ITO) branches, switching among three states.

between the transparent and mirror states at $\lambda=700 \mathrm{~nm}$ and the saturation current from the transparent and mirror states (Supplementary Figure S4). We measured transmittance changes at $\lambda=600 \mathrm{~nm}$ over 10000 cycles. In the $\mathrm{ECD}_{\text {ref }}$ (Figure $6 \mathrm{~b}$ ), transmittance changed arithmetically by $\sim 1 \%$ in the transparent state (first cycle $\sim 83.16 \%$ and 10000 th cycle $\sim 82.37 \%$ ) and by $\sim 4 \%$ in the mirror state (first cycle $\sim 9.99 \%$ and 10000 th cycle $\sim 13.96 \%$ ). The $\mathrm{ECD}_{\text {ref }}$ could not operate in the black state. In the $\mathrm{ECD}_{700}$ (Figure $6 \mathrm{c}$ ), the transmittance changed by $\sim 2 \%$ in the transparent state (first cycle $\sim 73.58 \%$ and 10000 th cycle $\sim 75.57 \%$ ), by $\sim 4 \%$ in the mirror state (first cycle $\sim 11.09 \%$ and 10000 th cycle $\sim 15.7 \%$ ) and by $\sim 17 \%$ in the black state (first cycle $\sim 10.62 \%$ and 10000 th cycle $\sim 27.53 \%$ ). Therefore, the $\mathrm{ECD}_{\text {ref }}$ is more stable than the $\mathrm{ECD}_{700}$, but it operates only in the transparent, mirror and mirror-like states (see Figure $3 \mathrm{~b}$ ) that have a nonblack color. The degradation of transmittance in the black state of the $\mathrm{ECD}_{700}$ is mainly because of the weak redox reaction of Ag NPs at the surface of the ITO branches that have a large surface area compared with the flat ITO. This problem could be improved by increasing the redox reaction time in the black state.

\section{CONCLUSION}

We demonstrated a novel Ag-ECD that used 3D nanostructured ITO branches to enhance LSPR. As $t_{\text {ITO increased, a branched }}$ hierarchical structure developed and became crosslinked. Ag NPs aggregated on the 3D ITO hierarchical structure can be spatially separated, and thus the reflection can be reduced according to the strong LSPR. These properties enhanced the formation of a deep black state by completely suppressing the reflection of incident light. The $\mathrm{ECD}_{\text {ref }}$ had high $R_{\mathrm{avg}}>50 \%$, possibly as a result of the weak LSPR of the Ag NPs on the flat ITO. However, our ECD had $T_{\mathrm{avg}}=73.76 \%$ in the transparent state, $R_{\text {avg }}=79.77 \%$ in the mirror state and $R_{\text {avg }}=8.78 \%$ in the black state. Furthermore, the ECD with 3D ITO branches exhibited high stability after switching for 10000 cycles among the three states. Our ECD with high stability can effectively achieve three states: the mirror, black and transparent states. It used $\mathrm{Ag}^{+}$ions dissolved in the liquid electrolyte, independent of the oxidation under ambient conditions, compared with the metallic Ag in the liquid electrolyte. Therefore, we anticipate that ECDs with 3D ITO branches will be promising candidates for applications in converging technologies for energy-saving windows, multifunctional displays, solar-energy systems and smart window systems. In particular, the applications of windows and displays may lead to a decrease in energy consumption by reversibly switching the incoming radiation in response to changing environmental conditions.

\section{CONFLICT OF INTEREST}

The authors declare no conflict of interest.

\section{ACKNOWLEDGEMENTS}

This work was supported by Brain Korea 21 PLUS for Center for Creative Industrial Materials and by the National Research Foundation of Korea (NRF) Grants funded by the Korean Government (MSIP, NRF-2013R1A2A2A01069237 and NRF-2014H1A2A1021655, Global PhD. Fellowship Program). We also acknowledge Samsung Display Co., Ltd for financial support of this work.

Author contributions: J-LL supervised the project, analyzed all the data and prepared the manuscript. KRJ and IL conducted most of the experiments, including fabrication of the ECDs, analyzed the experimental and simulation data and prepared the manuscript of the paper. JYP conducted FDTD simulations. CSC and S-HC discussed the experiments.

1 Niklasson, G. A. \& Granqvist, C. G. Electrochromics for smart window: thin films of tungsten oxide and nickel oxide, and devices based on these. J. Mater. Chem. 17 127-156 (2007).

2 Gillaspie, D. T., Tenent, R. C. \& Dillon, A. C. Metal-oxide films for electrochromic applications: present technology and future directions. J. Mater. Chem. 20, 9585-9592 (2010).

3 Ou, J. Z., Balendhran, S., Field, M. R., McCulloch, D. G., Zoolfakar, A. S., Rani, R. A., Zhuiykov, S., O'Mullane, A. P. \& Kalantar-Zadeh, K. The anodized crystalline $\mathrm{WO}_{3}$ nanoporous network with enhanced electrochromic properties. Nanoscale 4, 5980-5988 (2012).

4 Yen, H.-J., Chen, C.-J. \& Liou, G.-S. Flexible multi-colored electrochromic and volatile polymer memory devices derived from starburst triarylamine-based electoactive polyimide. Adv. Funct. Mater. 23, 5307-5313 (2013).

5 Liu, K., Varghese, J., Gerasimov, J. Y., Polyakov, A. O., Shuai, M., Su, J., Chen, D., Zajaczkowski, W., Marcozzi, A., Pisula, W., Noheda, B., Palstra, T. T. M., Clark, N. A. \& Herrmann, A. Controlling the volatility of the written optical state in electrochromic DNA liquid crystals. Nat. Commun. 7, 11476 (2016)

6 Alamer, F. A., Otley, M. T., Ding, Y. \& Sotzing, G. A. Solid-state high-throughput screening for color tuning of electrochromic polymers. Adv. Mater. 25, 6256-6260 (2013).

7 Ma, D., Shi, G., Wang, H., Zhang, Q. \& Li, J. Morphology-tailored synthesis of vertically aligned $1 \mathrm{D} \mathrm{WO}$ nano-structure films for highly enhanced electrochromic performance. J. Mater. Chem. A 1, 684-691 (2013).

8 Granqvist, C.-G. Electrochromic materials: out of a niche. Nat. Mater. 5, 89-90 (2006).

9 Gillaspie, D., Norman, A., Tracy, C. E., Pitts, J. R., Lee, S.-H. \& Dillon, A. Nanocomposite counter electrode materials for electrochromic windows. J. Electrochem. Soc. 3, H328-H331 (2010).

10 Yamada, Y., Sasaki, H., Tajima, K., Okada, M. \& Yoshimura, K. Optical switching properties of switchable mirrors based on $\mathrm{Mg}$ alloyed with alkaline-earth metals. Sol. Energy Mater. So. Cells 99, 73-75 (2012).

11 Krebs, F. C. Electrochromic displays: the new black. Nat. Mater. 7, 766-767 (2008).

12 Kim, J., Ong, G. K., Wang, Y., LeBlanc, G., Williams, T. E., Mattox, T. M., Helms, B. A. \& Milliron, D. J. Nanocomposite architecture for rapid, spectrally-selective electrochromic modulation of solar transmittance. Nano Lett. 15, 5574-5579 (2015).

13 Thakur, V. K., Ding, G., Ma, J., Lee, P. S. \& Lu, X. Hybrid materials and polymer electrolytes for electrochromic device applications. Adv. Mater. 24, 4071-4096 (2012).

14 Beaujuge, P. M. \& Reynolds, J. R. Color control in $\pi$-conjugated organic polymers for use in electrochromic devices. Chem. Rev. 110, 268-320 (2010).

15 Nam, Y. S., Park, H., Magyar, A. P., Yun, D. S., Pollom, T. S. Jr \& Belcher, A. M. Virustemplated iridium oxide-gold hybrid nanowires for electrochromic application. Nanoscale 4, 3405-3409 (2012).

16 Baetens, R., Jelle, B. P. \& Gustavsen, A. Properties, requirements and possibilities of smart windows for dynamic daylight and solar energy control in buildings: a state-of-theart-review. Sol. Energy Mater. So. Cells 94, 87-105 (2010).

17 Yao, D. D., Field, M. R., O'Mullane, A. P., Kalantar-Zadeh, K. \& Ou, J. Z. Electrochromic properties of $\mathrm{TiO}_{2}$ nanotubes coated with electrodeposited $\mathrm{MoO}_{3}$. Nanoscale 5, 10353-10359 (2013).

18 Zoombelt, A. P., Mathijssen, S. G. J., Turbiez, M. G. R., Wienk, M. M. \& Janssen, R. A. J. Small band gap polymers based on diketopyrrolopyrrole. J. Mater. Chem. 20, 2240-2246 (2010).

19 Araki, S., Nakamura, K., Kobayashi, K., Tsuboi, A. \& Kobayashi, N. Electrochromic optical-modulation device with reversible transformation between transparent, mirror, and black. Adv. Mater. 24, OP122-OP126 (2012).

20 Onodera, R., Seki, Y., Seki, S., Yamada, K., Sawada, Y. \& Uchida, T. Smart windows, switchable between transparent, mirror, and black states, fabricated using rough and smooth indium tin oxide films deposited by spray chemical vapor deposition. Appl. Phys. Express 6, 026503 (2013).

21 Park, C., Seo, S., Shin, H., Sarwade, B. D., Na, J. \& Kim, E. Switchable silver mirrors with long memory effects. Chem. Sci. 6, 596-602 (2015).

22 Willets, K. A. \& Van Duyne, R. P. Localized surface plasmon resonance spectroscopy and sensing. Annu. Rev. Phys. Chem. 58, 267-297 (2007).

23 Barnes, W. L., Dereux, A. \& Ebbesen, T. W. Surface plasmon subwavelength optics. Nature 424, 824-830 (2003). 
24 Scholl, J. A., Koh, A. L. \& Dionne, J. A. Quantum plasmon resonances of individual metallic nanoparticles. Nature 483, 421-427 (2012).

25 Liu, Y. \& Huang, C. Z. Screening sensitive nanosensors via the investigation of shapedependent localized surface plasmon resonance of single Ag nanoparticles. Nanoscale 5, 7458-7466 (2013).

26 Lee, I., Park, J. Y., Hong, K., Son, J. H., Kim, J. \& Lee, J.-L. The effect of localized surface plasmon resonance on the emission color change in organic light emitting diodes. Nanoscale 8, 6463-6467 (2016).

27 Livage, J. \& Ganguli, D. Sol-gel electrochromic coatings and devices: a review. Sol. Energy Mater. So. Cells 68, 365-381 (2001).

28 Baker, A. T., Bosi, S. G., Bell, J. M., MacFarlane, D. R., Monsma, B. G., Skryabin, I. \& Wang, J. Degradation mechanisms in electrochromic devices based on sol-gel deposited thin films. Sol. Energy Mater. So. Cells 39, 133-143 (1995).

29 Atwater, H. A. \& Polman, A. Plasmonics for improved photovoltaic devices. Nat. Mater 9, 205-213 (2010).

30 Park, S.-G., Mun, C., Lee, M., Jeon, T. Y., Shim, H.-S., Lee, Y.-J., Kwon, J.-D., Kim, C. S. \& Kim, D.-H. 3D hybrid plasmonic nanomaterials for highly efficient optical absorbers and sensor. Adv. Mater. 27, 4290-4295 (2015).

31 Yu, H. K., Kim, S., Koo, B., Jung, G. H., Lee, B., Ham, J. \& Lee, J.-L. Nano-branched transparent conducting oxides: beyond the brittleness limit for flexible electrode applications. Nanoscale 4, 6831-6834 (2012).

$32 \mathrm{Yu}$, H. K. \& Lee, J.-L. Growth mechanism of metal-oxide nanowires synthesized by electron beam evaporation: a self-catalytic vapor-liquid-solid process. Sci. Rep. 4, 6589 (2014)

33 Nguyen, P., Ng, H. T., Kong, J., Cassell, A. M., Quinn, R., Li, J., Han, J., McNeil, M. \& Meyyappan, M. Epitaxial directional growth of indium-doped tin oxide nanowire arrays. Nano Lett. 3, 925-928 (2003).

$34 \mathrm{Bu}$, X., Chen, H., Gai, H., Yang, R. \& Yeung, E. S. Scattering imaging of single quantum dots with dark-field microscopy. Anal. Chem. 81, 7507-7509 (2009).
35 Chen, T. \& Reinhard, B. M. Assembling color on the nanoscale: multichromatic switchable pixels from plasmonic atoms and molecules. Adv. Mater. 28, 3522-3527 (2016).

36 Hong, K., Son, J. H., Kim, K., Kim, S., Lee, I. \& Lee, J.-L. Modulation of surface plasmons coupling for enhancement of optical transmittance of silver-coated alkalineearth metal films. J. Mater. Chem. 22, 22859-22865 (2012).

37 Michaels, A. M., Nirmal, M. \& Brus, L. E. Surface enhanced Raman spectroscopy of individual rhodamine $6 \mathrm{G}$ molecules on large Ag nanocrystals. J. Am. Chem. Soc. 121, 9932-9939 (1999)

38 Wei, H., Reyes-Coronado, A., Nordlander, P., Aizpurua, J. \& Xu, H. Multipolar plasmon resonances in individual ag nanorice. ACS Nano 4, 2649-2654 (2010).

39 Zhou, J., Duan, B., Fang, Z., Song, J., Wang, C., Messersmith, P. B. \& Duan, H. Interfacial assembly of mussel-inspired au@ag@ polydopamine core-shell nanoparticles for recyclable nanocatalysts. Adv. Mater. 26, 701-705 (2014).

(c) (i) This work is licensed under a Creative Commons Attribution 4.0 International License. The images or other third party material in this article are included in the article's Creative Commons license, unless indicated otherwise in the credit line; if the material is not included under the Creative Commons license, users will need to obtain permission from the license holder to reproduce the material. To view a copy of this license, visit http:// creativecommons.org/licenses/by/4.0/

(C) The Author(s) 2017

Supplementary Information accompanies the paper on the NPG Asia Materials website (http://www.nature.com/am) 